\title{
Original
}

\section{Analysis of TEM-type Genes Derived from Cefotaxime-resistant Serratia marcescens}

\author{
Sadanori Kubo ${ }^{1)}$, Eihuu OGawa ${ }^{1)}$, Gelin $\mathrm{C}_{\text {hen }}{ }^{1}$, \\ Rika W $_{\text {AKUTA }}{ }^{2)}$ Kunihiko FUKUCHI ${ }^{11}$, Yasushi TAKAGI $^{1}$ \\ and Kunihide Gomi ${ }^{1 \prime}$
}

\begin{abstract}
We investigated the TEM-type extended spectrum beta-lactamases (ESBLs) in cefotaxime-resistant $S$. marcescens strains by means of a 24-month retrospective study between October 1996 and September 1998 at Showa University Hospital. Among the 579 S. marcescens strains isolated during this period, 51 were resistant to cefotaxime. We tested the expression of ESBLs in the cefotaxime-resistant strains using the disk susceptibility test with cefotaxime and cefotaxime/clavulanic acid. We then amplified the betalactamase gene in each strain by the polymerase chain reaction (PCR).

The inhibition diameters observed with cefotaxime and cefotaxime plus clavulanic acid were similar in all of the resistant strains, suggesting that the cefotaxime-resistant $S$. marcescens strains do not express ESBLs. Sequence analysis of the PCR-amplified beta-lactamase gene from clinical isolates of the cefotaxime-resistant strains identified it as TEM-1b. We detected the TEM-type beta-lactamase genes, TEM-1a and TEM-1b, in four cefotaximesensitive strains. In the TEM-1a-like gene of a cefotaxime-sensitive strain, we found one base substitution at nucleotide position 753 ( $\mathrm{C}$ to $\mathrm{A}$ ) causing an amino acid substitution from alanine 184 to valine. No ESBL TEM enzymes were found among these strains, but we isolated a novel TEM-type enzyme.

The cefotaxime-resistant $S$. marcescens isolates, like many gram-negative enterobacteria, had the TEM-1b gene of beta-lactamase. Resistance to cefotaxime seemed to have been acquired through other ESBLs, cephalosporinases or carbapenemases, and not through TEM-type ESBLs.
\end{abstract}

Key words : TEM, ESBLs, penicillinases, Serratia marcescens, cefotaxime

\section{Introduction}

Class A beta-lactamases such as TEM-type beta-lactamases, which are penicillinases transferred by plasmids, have been detected in enterobacteria since the 1960 's ${ }^{1}$. The excessive use of third-generation cephalosporins since the 1980's, mainly in Europe, has led to the development of extended-spectrum beta-lactamases (ESBLs) derived from class A beta-lactamases $^{2)}$. Novel ESBLs have since developed worldwide including in Japan. These enzymes are class A beta-lactamases according to the Bush-Jacoby-Mederiros classification,

1) Department of Clinical Pathology, Showa University School of Medicine, 1-5-8 Hatanodai, Shinagawa-ku, Tokyo 142-8555, Japan.

2) Central Clinical Laboratories, Showa University Hospital. 
and are defined as being inhibited by clavulanic acid ${ }^{3)}$. The relative hydrolytic efficacy of an ESBL should exceed $10 \%$ against cefotaxime, ceftazidime or aztreonam, assuming that the ESBL has $100 \%$ efficacy against penicillin $\mathrm{G}^{3)}$. In many countries including France, the U.S. and Italy, strains of the enterobacterium Serratia marcescens ( $S$. marcescens) that produce ESBLs have recently appeared ${ }^{2)}$.

We have investigated $S$. marcescens strains that are resistant to antibiotics in patients at our hospital. Here, we describe the results of genetic analyses of $S$. marcescens strains that are resistant to third-generation cephalosporins, to determine whether or not the resistance was derived from TEM-type ESBLs.

\section{Materials and Methods}

\section{Bacterial strains and culture}

Fifty-one clinical isolates of cefotaxime-resistant $S$. marcescens strains were analyzed. These strains were identified from the 579 strains of $S$. marcescens isolated from patients seen at Showa University Hospital between October 1996 and September 1998. A S. marcescens strain was considered to be resistant to cefotaxime as measured by the sensitivity test using MIC 2000 (Dynatec, Virginia, USA) ${ }^{4}$. Each strain was derived from a different patient.

To confirm whether each $S$. marcescens strain produced ESBLs, we followed the guidelines of the National Committee for Clinical Laboratory Standards (NCCLS) (M100-S9): an ESBL-producing strain was identified by the disk method using the KB disk (Eiken Kagaku, Tokyo, Japan) with $30 \mu \mathrm{g}$ cefotaxime or $30 \mu \mathrm{g} / 10 \mu \mathrm{g}$ cefotaxime/clavulanic acid on Mueller Hinton S agar medium (Eiken Kagaku, Tokyo, Japan); a strain showing an inhibition circle whose diameter was at least $5 \mathrm{~mm}$ larger after the addition of clavulanic acid, was considered to be an ESBL-producing strain $^{5)}$.

Polymerase chain reaction $(P C R)$

Three sets of original primers that recognize the common sequence of the TEM-type beta-lactamase gene, were synthesized to amplify nearly the entire TEM-type beta-lactamase gene.

1. Forward primer-1: 5'-GATCTCAACAGCGGTAAGATCC-3' Reverse primer-1 : 5'TCACTCATGGTTATGGCAGC-3' (Nucleotide positions $350-594$, PCR product $254 \mathrm{bp}$ )

2. Forward primer-2 : 5'-TATGCAGTGCTGCCATAACC-3' Reverse primer-2 : 5'-CGTTCATCCATAGTTGCCTG-3' (Nucleotide positions $567-1020$, PCR product 454 bp)

3. Forward primer-3 : 5'-TATGCAGTGCTGCCATAACC-3' Reverse primer-3 : 5'-CCAGTGCTGCAATGATACC-3' (Nucleotide positions 567-950, PCR product $384 \mathrm{bp}$ )

DNA from the clinically isolated bacteria was extracted using SepaGene (Sanko Junyaku, Tokyo, Japan). The PCR reaction (total 25 $\mu$ l) was performed with the Gene Amp PCR System 9600-R (Perkin-Elmer, Norwalk, CT, USA). The PCR reaction mixture contained $100 \mathrm{ng}$ of template DNA, 20 pmol of each primer, $200 \mu \mathrm{mol}$ of each dNTP, 1 unit of Taq DNA polymerase (Roche, Basel, Switzerland), and PCR reaction buffer ( $+\mathbf{M g}$ ) (Roche, Basel, Switzerland). The reaction conditions for primer set 1 were : $94^{\circ} \mathrm{C}$ for $30 \mathrm{sec}, 60^{\circ} \mathrm{C}$ for $90 \mathrm{sec}, 72^{\circ} \mathrm{C}$ for $90 \mathrm{sec}$, for 30 cycles. The reaction conditions for each of primer sets 2 and 3 were $94^{\circ} \mathrm{C}$ for $60 \mathrm{sec}, 60^{\circ} \mathrm{C}$ for $60 \mathrm{sec}$, and $72^{\circ} \mathrm{C}$ for $120 \mathrm{sec}$, for 30 cycles. The PCR product was separated by polyacrylamide gel electrophoresis (PAGE) on a $5 \%$ 
polyacrylamide gel.

\section{DNA sequencing}

PCR products were cloned into the pGEM-T Easy ${ }^{\mathrm{TM}}$ vector using the TA cloning method according to the manufacturer's instructions (Promega, Madison, WI, USA). The cloning site is located inside the beta-galactosidase coding region. After transfection into JM109, the insert DNA-containing pGEM vector-transfectants were isolated as white colonies on a Luria base agar plate containing X-gal (5-bromo-4-chloro-3-indolyl-beta-galactoside) and IPTG (isopropyl-beta-D-thiogalactopyranoside). The plasmid DNA was extracted from each clone by the alkaline sodium dodecyl sulfate (SDS) method $^{6)}$. This DNA was then digested with EcoRI, and the presence of the insert DNA was confirmed by $5 \%$ PAGE. After linearization with SalI, the plasmid DNA was sequenced with the A.L.F. DNA Sequencer II (Amersham Pharmacia, Uppsala, Sweden) using a fluorescent-labeled M13 forward sequencing primer (F5'CGACGTTGTAAAACGACGACGGCCAGT3') and the Thermo Sequenase Fluorescent Labeled Primer Cycle Sequencing kit (Amersham Pharmacia, Uppsala, Sweden) as described previously ${ }^{7)}$.

The position of the sequence was estimated from the position of the ampicillin resistance gene (TEM-1) as described by Sutcliffe ${ }^{8)}$.

\section{Results}

\section{Cefotaxime-resistant S. marcescens}

Fig. 1 shows the proportion of cefotaxime-resistant $S$. marcescens isolates identified from

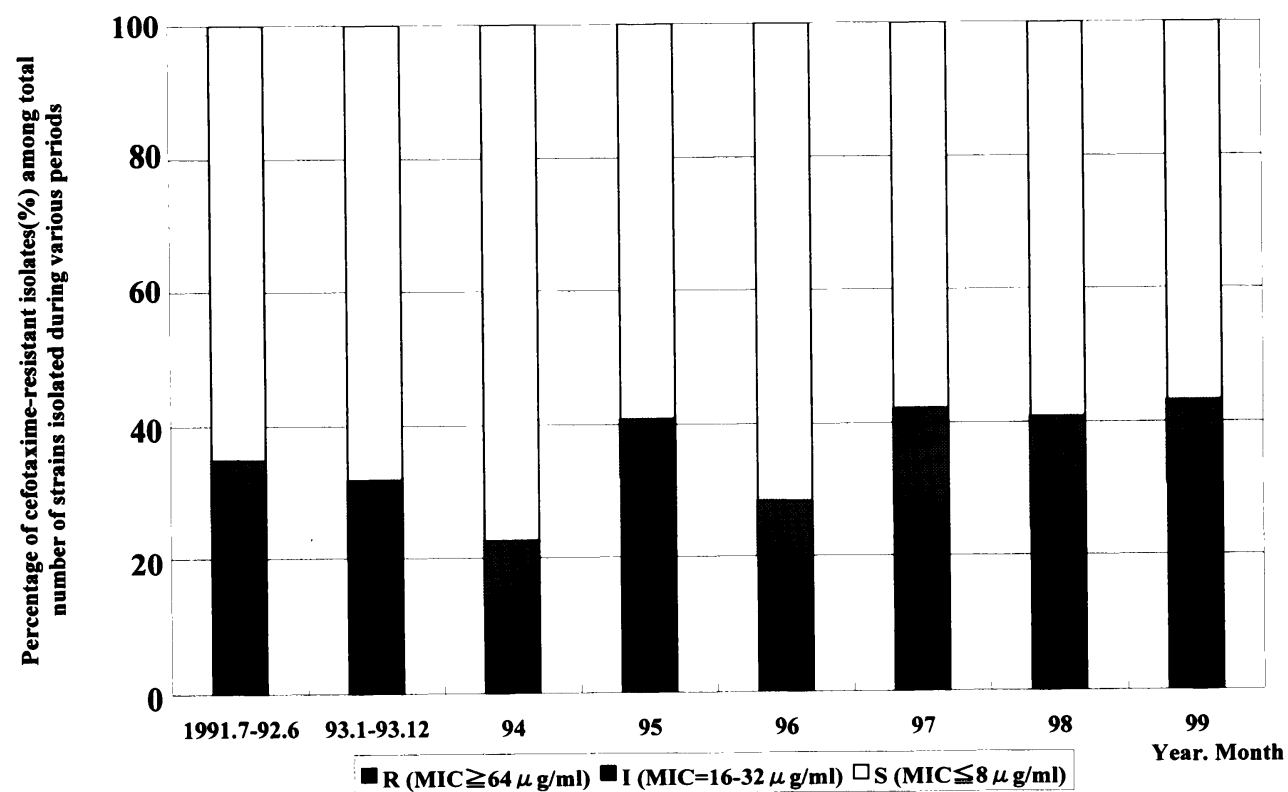

Fig. 1. Percentage of cefotaxime-resistant isolates among Serratia marcescens strains isolated during indicated time periods between 1991 and 1999 at Showa University hospital. Black bars represent proportion of resistant strains isolated during the indicated time period.

$\mathrm{S}$, susceptible ; I, intermediate ; $\mathbf{R}$, resistant. 
the total number of $S$. marcescens strains isolated at Showa University Hospital during the indicated periods between July 1991 through 1999. The percentage of cefotaxime-resistant S. marcescens is approximately $25-35 \%$ for each time period without any statistically significant difference.

\section{PCR analysis of the TEM-gene and inhibition by clavulanic acid}

Between October 1996 and September 1998, cefotaxime-resistant S. marcescens strains had been isolated from 51 patients. The patient characteristics and the departments in which they were treated are shown in Table 1. The minimum inhibitory concentration (MIC) value measured by the serial dilution method against major antibiotics is also shown. Whether the TEM-type beta-lactamase gene in each strain could be amplified with primer set 1,2 or 3 , is also shown in Table 1 . Based on the definition given by Bush et al. ${ }^{3)}$, that the resistance of ESBL-producing strains against third-generation cephalosporins is inhibited by clavulanic acid, the disk method was employed using the KB disk, which contains $30 \mu \mathrm{g}$ cefotaxime or $30 \mu \mathrm{g} / 10 \mu \mathrm{g}$ cefotaxime/clavulanic acid on Mueller Hinton S agar medium. The results of this test are also shown in Table 1.

There was no noteworthy feature among the cefotaxime-resistant strains except that they were mainly isolated from urine and respiratory samples. All of the strains showed resistance to ampicillin and cefotiam. All of the imipenem-resistant strains had been derived from urine samples. Primer sets 1, 2 and 3 could amplify DNA fragments of a TEM-type beta-lactamase gene in 30 of the 51 cefotaxime-resistant strains. This indicates that some cefotaxime-resistant strains have TEM-type genes. In the ESBL recognition test that we performed, none of the 51 strains completely satisfied the definition of the presence of an ESBL. However, in some strains there was enlargement of the inhibition circle by less than $5 \mathrm{~mm}^{3,5)}$.

\section{Sequencing of the TEM-gene}

As described above, PCR could amplify a TEM-type beta-lactamase gene in 30 of the 51 cefotaxime-resistant strains. Genetic analyses of the PCR products from these 30 strains revealed that all of the corresponding PCR products had the same sequence. The amplified products contained a C-to-T transition at nucleotide position 436 and a G-to-T transversion at position 604 compared with the sequence of the TEM-1a gene ${ }^{8)}$, and it was identical to the TEM-1b gene ${ }^{9,10)}$ which encodes penicillinase. The TEM-type ESBL gene was not identified (Fig. 2). Amino acids 21, 39 and 42 are substituted in various TEM derivatives, but since PCR primer set 1 starts at nucleotide 350 (amino acid number 47), we could not detect mutations occurring in the first 46 amino acids. Therefore, our sequencing data is incomplete (Fig. 2).

\section{TEM gene of four cefotaxime-susceptible strains}

Based on the data from PCR and sequencing, a TEM-type ESBL does not seem to be involved in the development of resistance to cefotaxime. Considering that the TEM-type genes obtained from the resistant strains encoded penicillinase, we next analyzed four $S$. marcescens strains that were sensitive to cefotaxime using the same procedure described above.

PCR analyses performed under the same conditions revealed a TEM-type gene in two of 
the four cefotaxime-sensitive strains (strains A and B), that could be amplified with primer sets 1 and 3 but not with primer set 2 (Table 1 ).

\section{Novel sequence of a TEM gene in cefotaxime-sensitive strains}

Genetic analysis of the PCR products from strain A that had been obtained with primer set 1 , revealed a sequence that was identical to the TEM-1a gene in three of the four clones of strain A. The fourth clone had a sequence that was identical to the TEM-1b or 1c gene, in which there was a mutation at position 436, a C-to-T transition, compared with the sequence of the TEM-1a gene (Fig. 2). With primer set 3, seven out of 12 clones showed an identical sequence with the TEM-1a gene except for a C-to-T transition at position 753, resulting in an alanine-to-valine substitution at amino acid 184. The remaining five clones had the TEM-1b gene with a C-to-T transition at position 436 and G-to-T transversion at position 604. The PCR products obtained from strain $B$ with primer sets 1 and 3 were identical to the TEM-1b gene (Fig. 2). The alanine-to-valine amino acid substitution at 184 was assumed to have no influence on protein structure, and was thought to represent a polymorphism rather than a mutation. The encoded protein would therefore function as a beta-lactamase. The MIC data are consistent with this assumption (Table 1).

\section{Discussion}

The number of $S$. marcescens strains isolated from patients seen at our hospital is increasing every year. Most $S$. marcescens strains are isolated from respiratory specimens, followed by urine specimens ${ }^{13)}$. The number of cefotaxime-resistant $S$. marcescens strains is also increasing. Most of the cefotaxime-resistant $S$. marcescens were isolated from urine specimens. This finding seems to represent a feature of most antibiotics that are excreted from the urinary tract. Because the urinary tract is exposed to high concentrations of antibiotics, prophlogistic bacteria are likely to acquire resistance and remain after screening.

ESBLs that can hydrolyze third-generation cephalosporin and aztreonam are considered to acquire the ability to hydrolyze novel antibiotics by mutation of the TEM-1a, TEM-1b, TEM-1c or TEM-2 genes, which code for plasmid-mediated penicillinases. The resulting amino acid substitutions are accompanied by a conformational change at the substratebinding site $^{3,11,12)}$. Most of the cefotaxime-resistant $S$. marcescens strains of interest contained a TEM-type gene. However, this TEM-type gene was the TEM-1b gene that encodes TEM-1 beta-lactamase. TEM-1 is a beta-lactamase that is commonly isolated from S. marcescens $^{14-16)}$. The TEM-1 beta-lactamase analyzed here seemed to be plasmid-mediated penicillinase, which is frequently observed in gram-negative enterobacteria. Since this penicillinase did not hydrolyze third-generation cephalosporines, resistance to cefotaxime seems to be acquired by other genetic resistance systems and not by TEM-type ESBLs.

The resistance of the cefotaxime-resistant strains isolated from the patients in this study may have been derived from a carbapenemase such as IMP-1, which is a class B metallo beta-lactamase ${ }^{17,18)}$. The IMP-1 gene is usually located on a plasmid, and IMP-1 confers resistance not only to carbapenem but also to a broad spectrum of beta-lactams including third-generation cephalosporins ${ }^{17-19}$. In fact, 11 of the 51 strains were resistant to imipenem, (MIC value of above $8 \mu \mathrm{g} / \mathrm{ml})^{4)}$, and these 11 strains showed strong and broad resistance to several beta-lactams including ceftazidime. PCR analysis using specific primers for the IMP-1 gene revealed that 8 of the 11 imipenem-resistant strains and 12 others contained the 


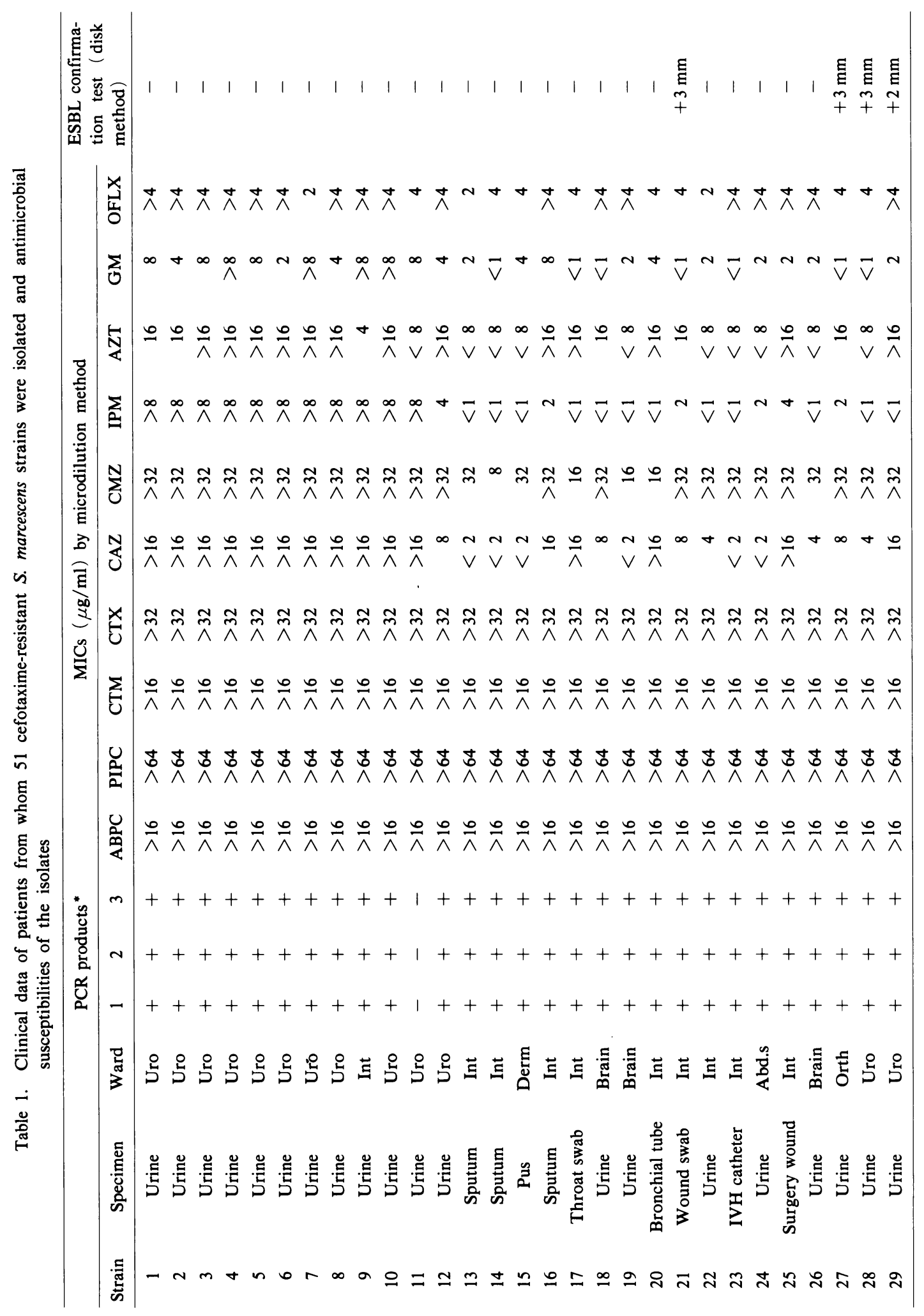




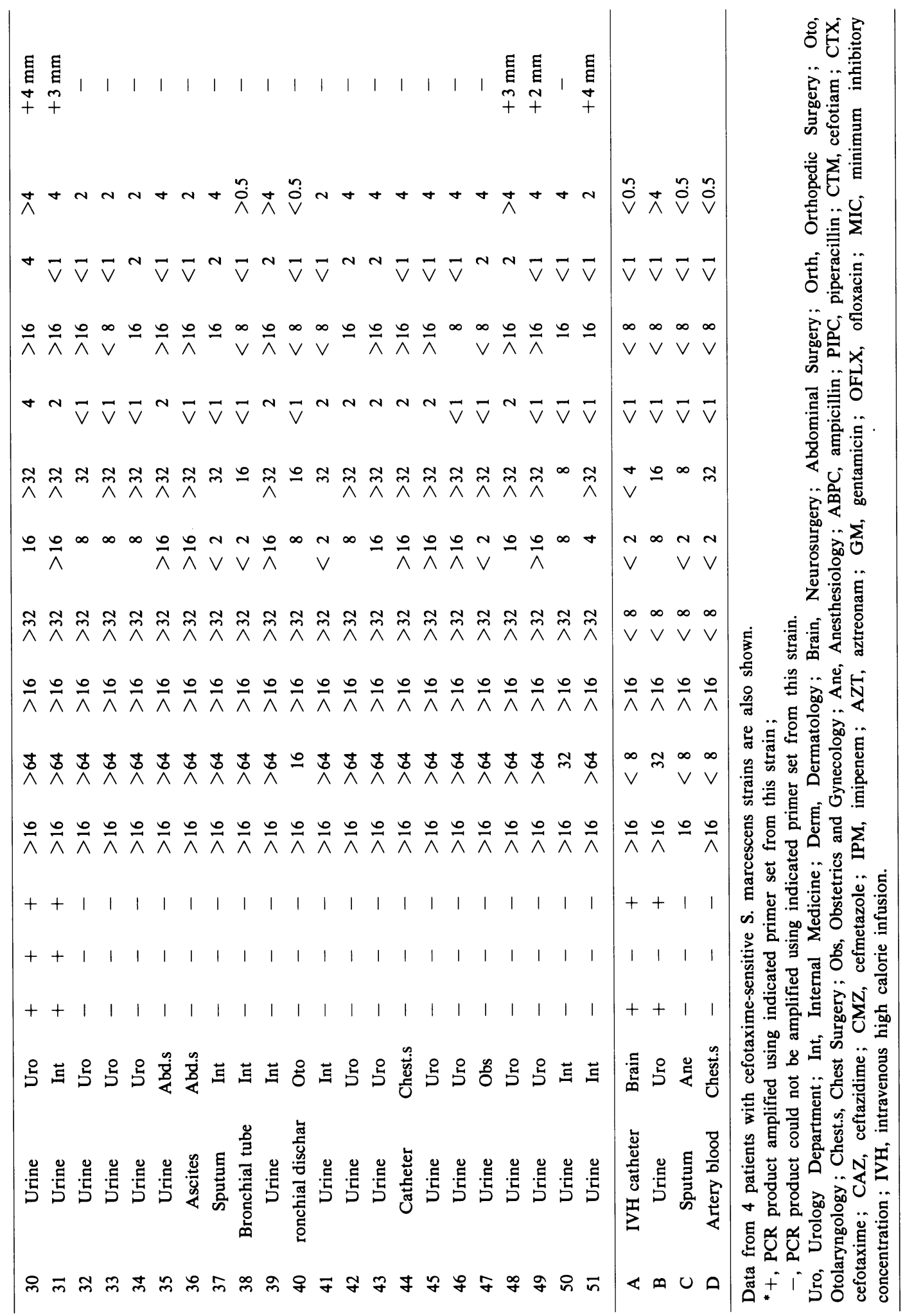




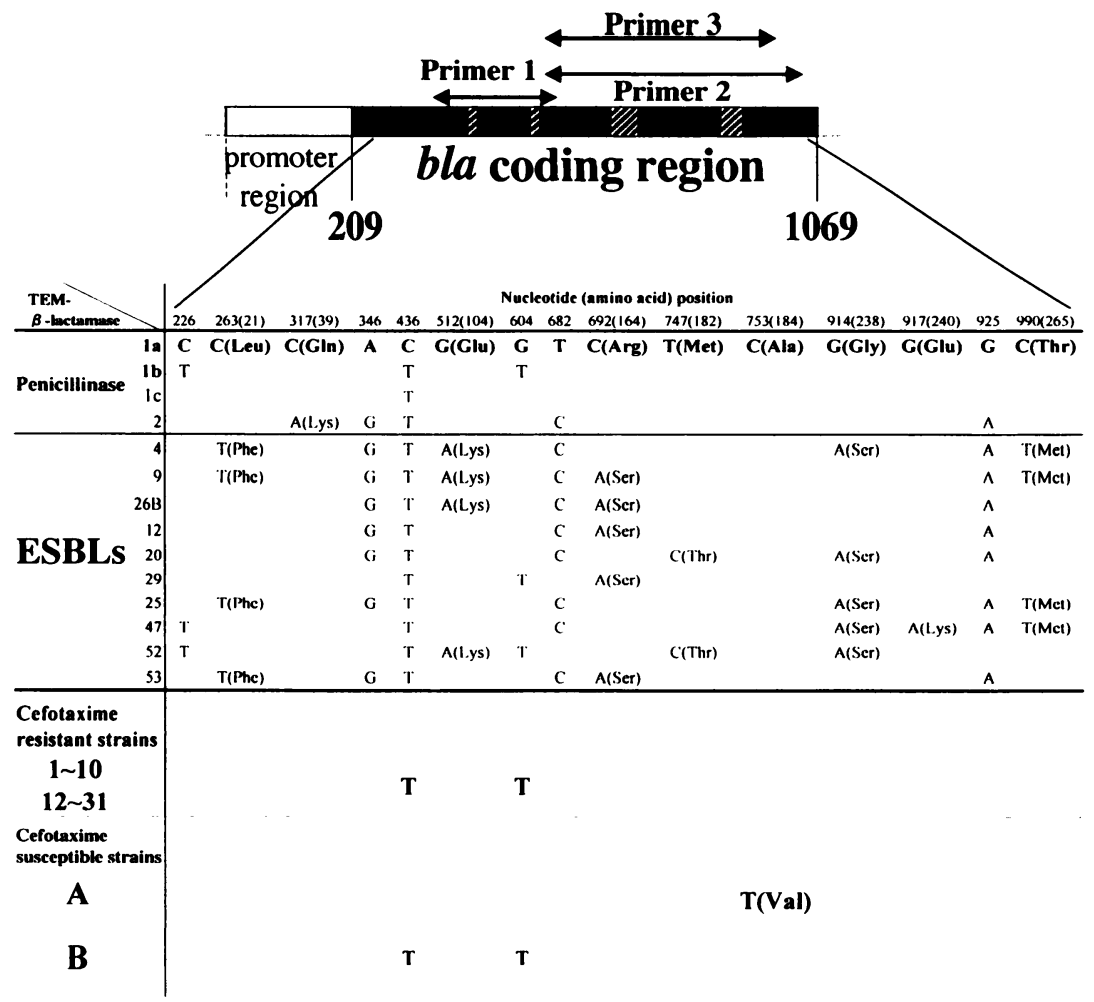

Fig. 2. Mutations in TEM-type beta-lactamase genes and experimental data. Only nucleotide

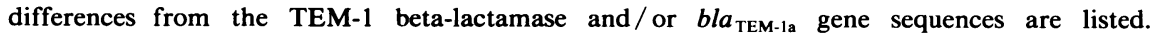
Hatched bars in the beta-lactamase coding region indicate the sequence encoding the beta-lactam binding site. Data are adapted from published reports ${ }^{8-12}$.

IMP-1 gene $^{20)}$. This observation also supports our hypothesis that the IMP-1 gene influences the resistance of the strains analyzed here.

Other than IMP-1, many mechanisms for the hydrolysis of third-generation cephalosporins in Enterobacteriaceae including $S$. marcescens, have been identified. For example, class C beta-lactamase is encoded by the cephalosporinase gene (ampC) located on the $S$. marcescens chromosome $^{21)}$. The degree of induction of beta-lactamase expression varies depending on the type of beta-lactam used: carbapenem and cephamycins are powerful inducers of beta-lactamase expression, while third-generation cephalosporins are weak inducers. Originally, beta-lactamase had no influence on third-generation cephalosporins. However, after a long period of usage of third-generation cephalosporins, a mutated strain that produces the enzyme appeared and proliferated ${ }^{22,23)}$. This third-generation cephalosporinresistant strain is only susceptible to carbapenem, which is stable against class $\mathrm{C}$ betalactamase. Therefore, ESBL inhibition by clavulanate would be masked by the presence of the AmpC enzyme that is present in all strains of Serratia. Furthermore, a number of our strains probably also possessed the IMP-1 beta-lactamase, which would mask the results of ESBL screening tests. 
Other forms of acquired third-generation cephalosporin resistance include the following: SHV-type ESBLs ${ }^{11,24}$, which constitute a class of ESBLs; non-TEM, non-SHV-type KIT-1 ${ }^{25)}$; CTX-M1 ${ }^{26)}$; CTX-M2 ${ }^{27)}$; TOHO- ${ }^{28)}$; and TOHO- ${ }^{29)}$. These and plasmid-derived cephalosporinase $^{30)}$ are assumed to be the mechanisms of resistance in most of the ESBL-producing strains isolated in Japan. Our clinically isolated strains may have been influenced by the resistance described above. Although we used cefotaxime-resistant strains in this study, these strains may not be appropriate to set the standard for the sensitivity to cefotaxime, because most ESBLs show a low MIC value towards cefotaxime ${ }^{4)}$ regardless of whether they show a high MIC score towards ceftazidime or aztreonam. This problem will be solved by using the ESBL Confirmation Panel (Dade Behring, CA, USA), which has recently become commercially available.

A TEM-1a-like gene with a C-to-T transition (184 alanine to valine) was detected in one of the four cefotaxime-susceptible strains. This mutation was not identical to any previously detected mutation site in various TEM-type ESBLs (Fig. 2). This one-base substitution was accompanied by an amino acid substitution ( 184 alanine to valine). However this strain was still susceptible to cefotaxime and the spectrum of susceptibility was not significantly different from that of frequently isolated $S$. marcescens strains. Taken together, we concluded that the TEM-1b gene isolated from the cefotaxime-resistant $S$. marcescens strains is not specific for these strains, but is commonly observed in $S$. marcescens strains.

The frequency with which cefotaxime-resistant $S$. marcescens is isolated at our hospital will likely should increase. This resistant property is not derived from TEM-type ESBLs since we detected the TEM-1b gene which produces TEM-1, a common penicillinase, in $S$. marcescens strains. In addition to the TEM-1b gene, which was also found in some of the sensitive strains, one strain carried a TEM-1a-like gene.

Few reports have described ESBL-producing strains in Japan, compared with European countries. To prevent an outbreak, the mechanism of resistance to beta-lactams must be elucidated, since these antibiotics play an important role in the therapy of infectious diseases.

\section{Acknowledgement}

Part of this study was supported by a grant-in-aid from the Ministry of Education, Science, Sports and Culture of Japan.

\section{References}

1) Datta $\mathbf{N}$ and Kontomichalou $\mathbf{P}$ : Penicillinase synthesis controlled by infectious $\mathbf{R}$ factors in Enterobacteriaceae. Nature 208 : 239-241 (1965)

2) Knothe H, Shah P, Krcmery V, Antal M and Mitsuhashi S : Transferable resistance to cefotaxime, cefoxitin, cefamandole and cefuroxime in clinical isolates of Klebsiella pneumoniae and Serratia marcescens. Infection 11 : 315-317 (1983)

3) Bush K, Jacoby GA and Medeiros AA: A functional classification scheme for beta-lactamases and its correlation with molecular structure. Antimicrob Agents Chemother 39 : 1211-1233 (1995)

4) National Committee for Clinical Laboratory Standards: Methods for dilution antimicrobial susceptibility test for bacteria that grow aerobically-fourth edition. Approved standard M7-A4, National Committee for Clinical Laboratory Standards, Villanova, Pa, (1997)

5) National Committee for Clinical Laboratory Standards: Performance standards for antimicrobial susceptibility testing. Ninth informational supplement M100-S9, National Committee for Clinical Laboratory Standards, Villanova, $\mathrm{Pa}$, (1999) 
6) Sambrook J, Fritsch EF and Maniatis T: Molecular Cloning: a Laboratory Manual. 2nd ed. Cold Spring Harbor Laboratory Press, New York, Cold Spring Harbor (1989)

7) Watanabe H, Fukuchi $\mathbf{K}$, Takagi Y, Tomoyasu S, Tsuruoka $\mathbf{N}$ and Gomi $\mathbf{K}$ : Molecular analysis of the Cip 1/Waf1 (p21) gene in diverse types of human tumors. Biochim Biophys Acta 1263 : 275-280 (1995)

8) Sutcliffe JG : Nucleotide sequence of the ampicillin resistance gene of Escherichia coli plasmid pBR322. Proc Natl Acad Sci USA 75 : 3737-3741 (1978)

9) Chen ST and Clowes RC: Variations between the nucleotide sequences of $\operatorname{Tn} 1, \operatorname{Tn} 2$, and $\operatorname{Tn} 3$ and expression of beta-lactamase in Pseudomonas aeruginosa and Escherichia coli. J Bacteriol 169 : 913-916 (1987)

10) Goussard $S$ and Courvalin $P$ : Sequence of the genes blaT-1B and blaT-2. Gene 102 : 71-73 (1991)

11) Gniadkowski M, Schneider I, Jungwirth R, Hryniewicz W and Bauernfeind A : Ceftazidime-resistant Enterobacteriaceae isolates from three Polish hospitals: identification of three novel TEM- and SHV-5-type extended-spectrum beta-lactamases. Antimicrob Agents Chemother 42 : 514-520 (1998)

12) Leflon-Guibout V, Speldooren V, Heym B and Nicolas-Chanoine MH: Epidemiological survey of amoxicillin-clavulanate resistance and corresponding molecular mechanisms in Escerichia coli isolates in France: New genetic features of blaTEM genes. Antimicrob Agents Chemother $44: 2709-2714$ (2000)

13) Kubo S, Kano M, Ogawa E, Maruyama H, Chen G, Wakuta R and Fukuchi K: Identification and susceptibility of clinically isolated bacteria. Statistical data at shown. University Hospital from 1997 and 1998 in comparison with an earlier period. J Showa Med Assoc 59 : 407-437 (1997) (in Japanese)

14) Bush K, Flamm RK, Ohringer S, Singer SB, Summerill $R$ and Bonner DP: Effect of clavulanic acid on activity of beta-lactam antibiotics in Serratia marcescens isolates producing both a TEM beta-lactamase and a chromosomal cephalosporinase. Antimicrob Agents Chemother 35 : 2203-2208 (1991)

15) Roy C, Fox A, Segura C, Tirado M, Fuster C and Reig R: Plasmid-determined beta-lactamases identified in a group of 204 ampicillin-resistant Enterobacteriaceae. J Antimicrob Chemother 12: $507-510$ (1983)

16) Yang YJ, Wu PJ and Livermore DM : Biochemical characterization of a beta-lactamase that hydrolyzes penems and carbapenems from two Serratia marcescens isolates. Antimicrob Agents Chemother 34 : 755-758 (1990)

17) Osano E, Arakawa Y, Wacharotayankun R, Ohta M, Horii T, Ito H, Yoshimura F and Kato N : Molecular characterization of an enterobacterial metallo beta-lactamase found in a clinical isolate of Serratia marcescens that shows imipenem resistance. Antimicrob Agents Chemother 38 : 71-78 (1994)

18) Rasmussen BA and Bush K : Carbapenem-hydrolyzing beta-lactamases. Antimicrob Agents Chemother 41 : $223-$ 232 (1997)

19) Arakawa Y, Murakami M, Suzuki K, Ito H, Wacharotayankun R, Ohsuka S, Kato N and Ohta M: A novel integron-like element carrying the metallo-beta-lactamase gene blaIMP. Antimicrob Agents Chemother 39 : $1612-1615$ (1995)

20) Ogawa E, Kubo S, Kanou M, Chen G, Wakuta R, Fukuchi K, Takagi Y and Gomi K: Detection of the blaIMP-1 beta-lactamase gene in clinically isolated cefotaxime-resistant strains of Serratia marcescens, Klebsiella pneumoniae, and Enterobacter cloacae. Showa Univ J Med Sci 13 : 69-78 (2001)

21) Lindberg F, Westman $\mathrm{L}$ and Normark $\mathrm{S}$ : Regulatory components in Citrobacter freundii ampC beta-lactamase induction. Proc Natl Acad Sci USA 82 : 4620-4624 (1985)

22) Satake S, Hiraoka $M$ and Mitsuhashi $S$ : Interaction of cefpirome and a cephalosporinase from Citrobacter freundii GN7391. Antimicrob Agents Chemother 33 : 398-399 (1989)

23) Satake S, Yoneyama $H$ and Nakae $T$ : Role of OmpD2 and chromosomal beta-lactamase in carbapenem resistance in clinical isolates of Pseudomonas aeruginosa. J Antimicrob Chemother 28 : 199-207 (1991)

24) Nordmann P: Trends in beta-lactam resistance among Enterobacteriaceae. Clin Infect Dis 27: S100-S106 (1998)

25) Inoue $M$, Fuse A, Yamaguchi K, Kanno H, Kaku M, Inamatsu T, Nagahama T, Yoshida M, Nonoyama M and Okamoto $\mathbf{R}$ : Characterization of an extended-spectrum beta-lactamase from Escherichia coli mediated by a plasmid. J Infect Chemother 1 : 70-72 (1995)

26) Bernard H, Tancrede C, Livrelli V, Morand A, Barthelemy M and Labia R: A novel plasmid-mediated extended-spectrum beta-lactamase not derived from TEM- or SHV-type enzymes. J Antimicrob Chemother 29 : 590-592 (1992)

27) Bauernfeind A, Stemplinger I, Jungwirth R, Ernst S and Casellas JM: Sequences of beta-lactamase genes encoding CTX-M-1 (MEN-1) and CTX-M-2 and relationship of their amino acid sequences with those of other beta-lactamases. Antimicrob Agents Chemother $40: 509-513$ (1996)

28) Ishii $\mathbf{Y}$, Ohno $A$, Taguchi $H$, Imajo $S$, Ishiguro $\mathbf{M}$ and Matsuzawa $\mathbf{H}$ : Cloning and sequence of the gene encoding a cefotaxime-hydrolyzing class A beta-lactamase isolated from Escherichia coli. Antimicrob Agents Chemother 39 : 2269-2275 (1995)

29) Ma L, Ishii $Y$, Ishiguro $M$, Matsuzawa $H$ and Yamaguchi $K$ : Cloning and sequencing of the gene encoding Toho-2, a class A beta-lactamase preferentially inhibited by tazobactam. Antimicrob Agents Chemother 42 : 
1181-1186 (1998)

30) Horii T, Arakawa Y, Ohta M, Sugiyama T, Wacharotayankun R, Ito $\mathbf{H}$ and Kato $\mathbf{N}$ : Characterization of a plasmid-borne and constitutively expressed blaMOX-1 gene encoding AmpC-type beta-lactamase. Gene 139: 93-98 (1994)

[Received June 27, 2001 : Accepted October 30, 2001] 